\title{
Pinning Controllability Scheme of Directed Complex Delayed Dynamical Networks via Periodically Intermittent Control
}

\author{
Shaolin $\mathrm{Li}^{1}{ }^{1}$ Jinde Cao, ${ }^{2}$ and Yinghui $\mathrm{He}^{1}$ \\ ${ }^{1}$ Department of Mathematics, Honghe University, Mengzi, Yunnan 661100, China \\ ${ }^{2}$ Department of Mathematics and Research Center for Complex Systems and Network Sciences, \\ Southeast University, Nanjing, Jiangsu 210096, China \\ Correspondence should be addressed to Jinde Cao; jdcao@seu.edu.cn
}

Received 29 August 2015; Revised 15 October 2015; Accepted 27 October 2015

Academic Editor: Daniele Fournier-Prunaret

Copyright (C) 2016 Shaolin Li et al. This is an open access article distributed under the Creative Commons Attribution License, which permits unrestricted use, distribution, and reproduction in any medium, provided the original work is properly cited.

\begin{abstract}
This paper studies the pinning controllability of directed complex delayed dynamical networks by using periodic intermittent control scheme. The general and low-dimensional pinning synchronization criteria are derived to illustrate the design of periodic intermittent control scheme. According to our low-dimensional pinning criterion, especially, the constraint condition of coupling strength is obtained when the network structure and amounts of pinned nodes are fixed. An algorithm is presented to determine the amounts of periodically intermittent controllers and locate these intermittent controllers in a directed network, in which the significance of nodes out- (in-) degree in pinning control of complex network is also illustrated. Finally, a directed network consisting of 12 coupled delayed Chua oscillators is designed as numerical example to verify the effectiveness of the theoretical analysis.
\end{abstract}

\section{Introduction}

Complex network has received increasing attention and has been intensively investigated in recent years due to its wide applications in many fields, such as the Internet, World Wide Web, social networks, citation network, and electrical power grids. The latest development of complex network focuses on the transition from a regular network to random network, in which the small-world and scale-free networks are mileposts in the field of complex network. Due to the pioneering work of Pecora and Carroll [1], synchronization and chaos control have received more and more attentions in various fields. Nowadays, chaotic synchronization has been applied widely in secure communication, chemical reactions, biological systems, and so on. Thus, plenty of scholars have devoted themselves to the study of convergence dynamics and proposed some novel concepts (e.g., globally exponential synchronization, lag synchronization, $H_{\infty}$ synchronization, and global quasi-synchronization) to describe the dynamical behavior of complex network [2-7].

In the case where the network cannot synchronize by itself, some appropriate controllers are inevitably required to regulate the network to a desired state. Correspondingly, many novel control techniques [8-11] including adaptive control, impulsive control, and switch control have been developed to force the network to realize synchronization. In practice, however, in a large-scale network with enormous nodes and links, it is too costly and infeasible to add controllers to all network nodes. Pinning control [12-17], in which controllers can be only applied to partial nodes, is used to reduce the amount of controllers. In [12], Yu et al. investigated some fundamental and challenging problems in pinning control of undirected complex network and pointed specially that the nodes with low degrees should be pinned firstly when the coupling strength is small. A concept known as pinning controllability [13] is defined to evaluate the performance of pinning control. In the works $[14,15]$ of Song et al., some low-dimensional pinning criteria and pinned-node selection scheme are proposed for global synchronization of both directed and undirected complex network. By $M$ matrix approach, they also discussed pinning controllability of complex network and designed a selective pinning scheme for a network with arbitrary topology to determine the amounts and the locations of the pinned nodes. Alternatively, 
intermittent control [18-22] can not only reduce the controlling cost, but also enhance the communication security. Therefore, intermittent control is an effective and widely used technique in engineering fields such as manufacturing and transportation. In [18], Xia and Cao considered a general model of complex delayed dynamical network and proposed some criteria for pinning synchronization of such dynamical network by periodically intermittent control method. However, the amounts and locations of the pinned nodes are not involved in their work. Cai et al. [20] investigated exponential synchronization for a class of complex delayed dynamical networks via pinning periodically intermittent control. They provided a pinning scheme deciding what nodes should be chosen as pinned candidates and how many nodes are needed to be pinned for a fixed coupling strength. However, it is regrettable that the proposed scheme is too simplified to be applied in practice.

Motivated by above novel results, we keep on studying pinning synchronization of a directed complex delayed network by utilizing the periodically intermittent control. In this paper, our attention is directed to delayed dynamical network and its synchronized criteria. The least number of pinned nodes can be determined under the given network configuration and coupling strength by the proposed synchronized criteria; on the other hand, the two constraints for coupling strength are provided by the network configuration and number of controllers. As a result, we propose a pinned-node selection algorithm, in which the amounts, type of pinned nodes, and feedback gain of controllers are determined in detail. Meanwhile, we point out especially that the node with zero in-degree must be selected as candidate.

The rest of this paper is organized as follows. In Section 2, some preliminaries and model description are stated briefly. In Section 3, some synchronization criteria for directed complex delayed dynamical network via periodically intermittent control are presented, in which the least number of pinned nodes and constraints for coupling strength are provided. In Section 4, an algorithm for selecting pinned nodes and designing feedback gain of controllers are proposed for a directed network. In Section 5, a numerical example is given to validate the effectiveness of theoretical result. Finally, conclusions are drawn in Section 6.

\section{Preliminaries and Model Description}

Throughout this paper, the following standard notations are used. $\mathbb{R}^{N}$ denotes the $N$-dimensional real Euclidean space. Let $\mathbb{R}^{N \times N}$ be $N \times N$ real matrices; let $I_{N} \in \mathbb{R}^{N \times N}$ be an $N$ dimensional identity matrix; let $\mathbf{1}_{N} \in \mathbb{R}^{N}$ be the vector of all ones. For a real matrix $A \in \mathbb{R}^{N \times N}, A^{T}$ is its transpose and $A^{s}=\left(A+A^{T}\right) / 2$ is its symmetric part; its inverse is denoted by $A^{-1}$; denote its $i$ th eigenvalue by $\lambda_{i}(A)$. For the matrix $G \in \mathbb{R}^{N \times N}$, we denote $(G)_{l} \in \mathbb{R}^{(N-l) \times(N-l)}$ by a minor matrix of $G$ by removing the $1,2, \ldots, l$ row-column pairs of matrix $G$. For real symmetric matrices $X$ and $Y$, the notation $X<Y(X \leq Y)$ means that $X-Y$ is negative definite (resp., negative semidefinite); $\lambda_{\text {min }}(X)$ and $\lambda_{\text {max }}(X)$ denote its minimum and maximum eigenvalue. $\operatorname{diag}(\cdots)$ stands for a block-diagonal matrix; $\otimes$ denotes the Kronecker product. The matrices are assumed to have compatible dimensions if they are not explicitly stated.

To derive our main results, the following lemmas are essential.

Lemma 1 (see [23]). Let $\omega:[\mu-\tau,+\infty) \rightarrow[0,+\infty)$ be a differentiable function and let $a, b$ be positive constants. If $a>b$ and $\dot{\omega}(t) \leq-a \omega(t)+b\left(\sup _{t-\tau \leq \theta \leq t} \omega(\theta)\right)$ are satisfied for $t \geq \mu$, then the following inequality

$$
\omega(t) \leq\left(\sup _{\mu-\tau \leq \theta \leq \mu} \omega(\theta)\right) \exp \{-\sigma(t-\mu)\}, \quad t \geq \mu,
$$

holds, where $\sigma>0$ is the smallest real root of the equation $a-z-b \exp \{z \tau\}=0$.

Lemma 2 (see [18]). Let $\omega:[\mu-\tau,+\infty) \rightarrow[0,+\infty)$ be a differentiable function and let $a, b$ be arbitrary positive constant. If the inequality $\dot{\omega}(t) \leq a \omega(t)+b\left(\sup _{t-\tau \leq \theta \leq t} \omega(\theta)\right)$ is satisfied for $t \geq \mu$, then $\omega(t) \leq\left(\sup _{\mu-\tau \leq \theta \leq \mu} \omega(\theta)\right) \exp \{(a+$ b) $(t-\mu)\}$.

Lemma 3 (see [14]). Suppose that $A, B$ are $n \times n$ Hermitian matrices. Let $\alpha_{1} \geq \alpha_{2} \geq \cdots \geq \alpha_{n}, \beta_{1} \geq \beta_{2} \geq \cdots \geq \beta_{n}$, and $\xi_{1} \geq \xi_{2} \geq \cdots \geq \xi_{n}$ be eigenvalues of $A, B$ and $A+B$, respectively. Then, one has $\alpha_{i}+\beta_{n} \leq \xi_{i} \leq \alpha_{i}+\beta_{1}, i=1,2, \ldots, n$.

This paper is devoted to a general delayed dynamical complex network consisting of $N$ identical nodes with linearly diffusive coupling, and each node is an $m$-dimensional dynamic system. The network can be described by

$$
\begin{array}{r}
\dot{x}_{i}(t)=f\left(t, x_{i}(t), x_{i}(t-\tau)\right)+c \sum_{j=1}^{N} g_{i j} \Gamma x_{j}(t), \\
\quad i=1,2, \ldots, N,
\end{array}
$$

where $x_{i}(t)=\left(x_{i 1}(t), x_{i 2}(t), \ldots, x_{i m}(t)\right)^{T}$ is the state variable of the $i$ th node, $\tau>0$ is the time delay, $c>0$ is the coupling strength, and $f\left(t, x_{i}(t), x_{i}(t-\tau)\right)=\left(f_{1}\left(t, x_{i}(t), x_{i}(t-\right.\right.$ $\left.\tau)), \ldots, f_{m}\left(t, x_{i}(t), x_{i}(t-\tau)\right)\right)^{T} \in \mathbb{R}^{m}$ is a continuous vector-valued function. The inner coupling matrix $\Gamma=$ $\operatorname{diag}\left(\gamma_{1}, \gamma_{2}, \ldots, \gamma_{m}\right)$ is a diagonal matrix with $\gamma_{i}>0$, and $G=\left(g_{i j}\right)_{N \times N}$ is the coupling configuration matrix describing topological structure of network, whose elements are defined as follows: for distinct nodes $i$ and $j$, if there is a directed link from node $j$ to node $i$, then $g_{i j}>0$; otherwise $g_{i j}=0$ and the diagonal element $g_{i i}=-\sum_{j=1, j \neq i}^{N} g_{i j}, i=1,2, \ldots, N$. It is noted that $\sum_{j=1}^{N} g_{i j}=0$. Furthermore, assume that the initial condition of the network is given by

$$
x_{i}(t)=\phi_{i}(t) \in \mathscr{C}\left([-\tau, 0], \mathbb{R}^{m}\right), \quad i=1,2, \ldots, N,
$$

where $\mathscr{C}\left([-\tau, 0], \mathbb{R}^{m}\right)$ denotes the set of continuous functions mapping $[-\tau, 0]$ into $\mathbb{R}^{m}$. 
Let $\operatorname{Deg}_{\text {in }}(i)$ and $\operatorname{Deg}_{\text {out }}(i)$ be the in-degree and outdegree of the $i$ th node. According to the definition of coupling configuration matrix $G$, it is easy to verify that

$$
\begin{aligned}
\operatorname{Deg}_{\text {in }}(i) & =-g_{i i}=\sum_{j=1, j \neq i}^{N} g_{i j}, \\
\operatorname{Deg}_{\text {out }}(i) & =\sum_{j=1, j \neq i}^{N} g_{j i},
\end{aligned}
$$

$$
i=1,2, \ldots, N \text {. }
$$

It should be noted that what type of node is selected as pinning candidates due to its degree information. The solution of an isolated node is described by

$$
\dot{s}(t)=f(t, s(t), s(t-\tau)),
$$

where $s(t)=\left(s_{1}(t), \ldots, s_{m}(t)\right)^{T} \in \mathbb{R}^{m}$. To realize the synchronization of network (2), the pinning control strategy is adopted if the network is not self-synchronized. We apply these control actions to a part of all nodes in the network. Suppose that nodes $i_{1}, i_{2}, \ldots, i_{l}(1 \leq l<N)$ in network are selected to be pinned. Without loss of generality, we can rearrange the order of the nodes in the network such that the first $l$ nodes are controlled. Now, we can get the pinning controlled network as follows:

$$
\begin{array}{r}
\dot{x}_{i}(t)=f\left(t, x_{i}(t), x_{i}(t-\tau)\right)+c \sum_{j=1}^{N} g_{i j} \Gamma x_{j}(t)+u_{i}(t), \\
i=1,2, \ldots, l, \\
\dot{x}_{i}(t)=f\left(t, x_{i}(t), x_{i}(t-\tau)\right)+c \sum_{j=1}^{N} g_{i j} \Gamma x_{j}(t), \\
i=l+1, \ldots, N .
\end{array}
$$

In most existing literatures, the feedback controllers are fulltime (e.g., $u_{i}=-d_{i} \Gamma\left(x_{i}(t)-s(t)\right), d_{i}>0, t>0$ ). In this paper, periodically intermittent control scheme is used and the intermittent controllers $u_{i}(i=1,2, \ldots, l)$ can be designed as follows:

$$
\begin{aligned}
& u_{i}(t) \\
& \quad= \begin{cases}-d_{i} \Gamma\left(x_{i}(t)-s(t)\right), & t \in[n T, n T+\delta), \\
0, & t \in[n T+\delta,(n+1) T),\end{cases}
\end{aligned}
$$

where the feedback gain $d_{i}>0, i=1,2, \ldots, l$, and control period $T>0,0<\delta<T, n=0,1,2, \ldots$.

The objective of control is to design the appropriate parameters $T, \delta$, and $d_{i}$ in controllers (7) such that the solutions of the controlled network (6) synchronize with the solution of (5), in the sense that

$$
\lim _{t \rightarrow \infty}\left\|x_{i}(t)-s(t)\right\|=0, \quad i=1,2, \ldots, N .
$$

Denoting the error vectors by $e_{i}(t)=x_{i}(t)-s(t),(1 \leq i \leq$ $N$ ), and subtracting (5) from (6), then the error dynamical network can be written as follows:

$$
\begin{aligned}
\dot{e}(t)= & F(t, x(t), x(t-\tau))-1_{N} \otimes f(t, s(t), s(t-\tau)) \\
& +((c G-D) \otimes \Gamma) e(t), \quad t \in[n T, n T+\delta), \\
\dot{e}(t)= & F(t, x(t), x(t-\tau))-1_{N} \otimes f(t, s(t), s(t-\tau)) \\
& +c(G \otimes \Gamma) e(t), \quad t \in[n T+\delta,(n+1) T),
\end{aligned}
$$

where $F(t, x(t), x(t-\tau))=\left(f^{T}\left(t, x_{1}(t), x_{1}(t-\tau)\right)\right.$, $\left.f^{T}\left(t, x_{2}(t), x_{2}(t-\tau)\right), \ldots, f^{T}\left(t, x_{N}(t), x_{N}(t-\tau)\right)\right)^{T}, e(t)=$ $\left(e_{1}^{T}(t), \ldots, e_{N}^{T}(t)\right)^{T}$ and $D=\operatorname{diag}\left(d_{1}, d_{2}, \ldots, d_{l}, 0, \ldots, 0\right) \epsilon$ $\mathbb{R}^{N \times N}$.

To derive the synchronized criteria for pinning controlled network (6), throughout the rest of this paper, the following assumptions are necessary.

Assumption 4. There exist two constant matrices $K=$ $\left(k_{i j}\right)_{m \times m}, W=\left(w_{i j}\right)_{m \times m} \in \mathbb{R}^{m \times m}$, such that the nonlinear function $f$ in network (6) satisfies

$$
\begin{aligned}
& \left|f_{i}(t, x(t), x(t-\tau))-f_{i}(t, y(t), y(t-\tau))\right| \\
& \quad \leq \sum_{j=1}^{m}\left(k_{i j}\left|x_{j}(t)-y_{j}(t)\right|\right. \\
& \left.\quad+w_{i j}\left|x_{j}(t-\tau)-y_{j}(t-\tau)\right|\right),
\end{aligned}
$$

where $x(t)=\left(x_{1}(t), \ldots, x_{m}(t)\right)^{T}, y(t)=\left(y_{1}(t), \ldots, y_{m}(t)\right)^{T} \epsilon$ $\mathbb{R}^{m}$.

According to Assumption 4, we introduce the following parameters:

$$
\begin{aligned}
& p=\max _{1 \leq r \leq m} \sum_{v=1}^{m}\left(k_{r v}^{2 \varepsilon}+k_{v r}^{2(1-\varepsilon)}+w_{r v}^{2 \varepsilon}\right), \\
& q=\max _{1 \leq r \leq m} \sum_{v=1}^{m} w_{v r}^{2(1-\varepsilon)},
\end{aligned}
$$

where $0 \leq \varepsilon \leq 1$.

Assumption 5. Denote that $V=\{1,2, \ldots, N\}$ and $V_{\text {pin }}=$ $\{1,2, \ldots, l\}$ are the sets of all nodes and pinned nodes for network (6), respectively. For any node $i \in V \backslash V_{\text {pin }}$, we can find at least a node $j \in V_{\text {pin }}$ such that there is a directed path from node $j$ to node $i$.

\section{Pinning Synchronization Criteria}

To realize the pinning synchronization of the controlled network (6), there are two crucial problems to be solved: (i) How many and what type of nodes should be chosen as pinned candidates? (ii) How may the intermittent controller's parameters $T, \delta$, and $d_{i}$ be designed suitably for each pinned node? To answer these questions, we state a general synchronization criterion for network (6). 
Theorem 6. Suppose that Assumptions 4 and 5 and $\tau \leq \delta$ and $\tau \leq T-\delta$ hold. Denote that $R_{1}=\delta / T, R_{2}=\tau / T$. If there exist positive constants $a, b$, and $d_{i}(i=1,2, \ldots, l)$, such that the following conditions are satisfied:

$$
\begin{aligned}
\frac{a+p}{2 \gamma} I_{N}-D+c G^{s} & <0, \\
a & >q, \\
\frac{p-b}{2 \gamma} I_{N}+c G^{s} & <0, \\
\sigma\left(R_{1}-R_{2}\right)-(b+q)\left(1-R_{1}\right) & >0,
\end{aligned}
$$

where $\gamma=\min _{1 \leq j \leq m} \gamma_{j}$ and $\sigma>0$ is the smallest real root of equation $a-z-q \exp (z \tau)=0$, then, the pinning controlled network (6) is globally synchronized with the solution of (5) under the periodically intermittent controllers (7).

Proof. The proof is given in Appendix.

It should be noted that the outer parameters $a$ and $b$ are introduced in Theorem 6 . The purpose for introducing constants $a$ and $b$ is based on the idea of linear matrix inequality (LMI) design. In Theorem 6, there are two LMIs involved in (12). It is well known that the introduction of some free parameters can improve greatly the solvability of LMI, although these parameters are not included in system. The effectiveness of the above idea and design had been proposed and validated in [18] and references therein.

For practical application, we are going to present some low-dimensional and convenient conditions to ensure global synchronization of the pinning process. Construct a symmetric matrix $H=\left(h_{i j}\right)_{N \times N}$ as follows:

$$
H=\frac{a+p}{2 \gamma} I_{N}+c G^{s}
$$

Denoting $\bar{D}=\operatorname{diag}\left(d_{1}, d_{2}, \ldots, d_{l}\right)$ and decomposing the matrix $H-D$, we have

$$
H-D=\left[\begin{array}{cc}
E-\bar{D} & B \\
B^{T} & C
\end{array}\right],
$$

where $E$ and $B$ are matrices with appropriate dimension. $C=$ $H_{l}$ is the minor matrix of $H$ by removing its first $l$ row-column pairs. According to Schur complement theorem, it is easy to confirm that $H-D<0$ is equivalent to

$$
E-\bar{D}-B C^{-1} B^{T}<0, \quad C<0 .
$$

According to the above analyses, we derive the following applicable corollary.

Corollary 7. Suppose that Assumptions 4 and 5 and $\tau \leq \delta$, $\tau \leq T-\delta$ hold; if there are positive real $a, b$, and $d_{i}$, then the pinning controlled network (6) is globally synchronized with the solution of (5) by periodically intermittent controller (7) when the following conditions are satisfied:

$$
\begin{aligned}
& d_{i}>\lambda_{\max }\left(E-B C^{-1} B^{T}\right), \quad i=1, \ldots, l, \\
& 2 c \gamma \lambda_{\max }\left(\left(G^{s}\right)_{l}\right)<-a-p, \\
& a>q, \\
& 2 c \gamma \lambda_{\max }\left(G^{s}\right)<b-p, \\
& \sigma\left(R_{1}-R_{2}\right)-(b+q)\left(1-R_{1}\right)>0,
\end{aligned}
$$

where $R_{1}, R_{2}, \sigma$, and $\gamma$ are totally the same as the ones in Theorem 6.

Proof. Note that $d_{i}>\lambda_{\max }\left(E-B C^{-1} B^{T}\right), i=1,2, \ldots, l$; it is verified that $E-\bar{D}-B C^{-1} B^{T}<0$ is satisfied. On the other hand, by Lemma 3, we have

$$
\begin{aligned}
\lambda_{\max }(C) & \leq \frac{a+p}{2 \gamma}+c \lambda_{\max }\left(\left(G^{s}\right)_{l}\right), \\
\lambda_{\max }\left(\frac{p-b}{2 \gamma} I_{N}+c G^{s}\right) & \leq \frac{p-b}{2 \gamma}+c \lambda_{\max }\left(G^{s}\right) .
\end{aligned}
$$

According to condition (16), it is easy to obtain that

$$
\begin{gathered}
\left(\frac{a+p}{2 \gamma} I_{N}+c G^{s}\right)_{l}<0, \\
\frac{p-b}{2 \gamma} I_{N}+c G^{s}<0 .
\end{gathered}
$$

By Theorem 6, we complete the proof.

In the case without time delayed term for network (6), that is, $\tau=0$, the periodically intermittent controllers (7) can also be adopted.

Remark 8. According to the processes of proving Theorem 6, it is inevitable that the network is coupled innerly through full state variable. Recall the definition of inner coupling matrix $\Gamma$; if the inner coupling matrix $\Gamma=\operatorname{diag}\left(\gamma_{1}, \ldots, \gamma_{m_{0}}, 0, \ldots, 0\right)$ with $\gamma_{j} \neq 0, j=1, \ldots, m_{0}$, then, it failed to apply the periodically intermittent controllers (6) to satisfy the sufficient conditions of Theorem 6 and Corollary 7. Thus, some other analysis approaches may be considered to solve this problem.

Remark 9. It is well known that the undirected network can be regarded as a directed network with completely symmetric coupling matrix, that is, $G=G^{T}$. In this case, it is easy to see that $G^{S}=G$. Hence, the sufficient conditions established in Theorem 6 and Corollary 7 for pinning synchronization are suitable for both directed and undirected complex network.

Remark 10. In Corollary 7, the first inequality of condition (16) provides a low bound of pinning feedback gains and can be used to design the pinning feedback gains. For the given network structure and coupling strength, the second inequality may be used to determine the least number $l_{0}$ of 
pinned nodes. Indeed, we need to find a positive integer $l_{0}$ such that the inequalities

$$
\begin{aligned}
\lambda_{\max }\left(\left(G^{s}\right)_{l_{0}-1}\right) & \geq-\frac{a+p}{2 c \gamma}, \\
\lambda_{\max }\left(\left(G^{s}\right)_{l_{0}}\right) & <-\frac{a+p}{2 c \gamma}
\end{aligned}
$$

hold simultaneously. On the other hand, for the fixed network structure and selected $l$ pinned nodes, if $\lambda_{\text {max }}\left(G^{s}\right)>0$, then the coupling strength required that

$$
-\frac{a+p}{2 \gamma \lambda_{\max }\left(\left(G^{s}\right)_{l}\right)}<c<\frac{b-p}{2 \gamma \lambda_{\max }\left(G^{s}\right)} .
$$

Otherwise, if $\lambda_{\max }\left(G^{s}\right)<0$, then the coupling strength is expected to satisfy

$$
c>\max \left\{-\frac{a+p}{2 \gamma \lambda_{\max }\left(\left(G^{s}\right)_{l}\right)}, \frac{b-p}{2 \gamma \lambda_{\max }\left(G^{s}\right)}\right\} .
$$

Usually, the theoretical coupling strength $c$ may be much larger than the needed value. To realize synchronization of network (6), the adaptive control approach may be adopted to tune online the coupling strength.

\section{Scheme for Selecting Pinned Candidates}

Up to this point, we have presented the pinning synchronous criteria for complex delayed network (6) with the periodically intermittent controllers (7). Theoretically, the conditions of Corollary 7 are more conservative than the ones in Theorem 6. However, using Corollary 7, we can give a guidance to design the periodically intermittent controllers and allocate these controllers in the network. In the following, the detailed procedure will be described.

For the fixed coupling strength and relevant configuration of network (6), we evaluate the parameters $p, q, \gamma$, and $\lambda_{\max }\left(G^{s}\right)$. Choose the appropriate parameters $a, b, T$, and $\delta$ and compute $R_{1}, R_{2}$, and $\sigma$ such that

$$
\begin{aligned}
a & >q, \\
\lambda_{\max }\left(G^{s}\right) & <\frac{b-p}{2 c \gamma}, \\
\sigma\left(R_{1}-R_{2}\right)-(b+q)\left(1-R_{1}\right) & >0
\end{aligned}
$$

is satisfied firstly.

In order to satisfy $\lambda_{\max }\left(\left(G^{s}\right)_{l}\right)<-(a+p) / 2 c \gamma$, we should choose some pinned candidates such that $\lambda_{\max }\left(\left(G^{s}\right)_{l}\right) \leq 0$. There is a widely concerned and discussed issue of what kind of node is selected as pinned candidate. In literature [14], authors have verified that if all nodes of network satisfy Assumption 5, $\operatorname{Deg}_{\text {out }}(i)>\operatorname{Deg}_{\text {in }}(i)(i=1,2, \ldots, l)$ and $\operatorname{Deg}_{\text {out }}(i) \leq \operatorname{Deg}_{\text {in }}(i)(i=l+1, \ldots, N)$. Then, $\lambda_{\text {max }}\left(\left(G^{s}\right)_{l}\right) \leq 0$ holds. Emulating the scheme of [14], we also rearrange all nodes in accordance with the sequence: the nodes with zero in-degree, the nodes with the diminishing difference values of out-degree and in-degree. For the nodes with same degree differences, we sort them with diminishing out-degree. We construct the coupling configuration matrix according to rearranged nodes and their inherent connections. Next, we get a simple algorithm to select pinned candidates using rearranged coupling configuration matrix. For simplicity, we still denote the rearranged coupling configuration matrix by $G$ and initialize $l=1$.

Step 1. Select the first $l$ nodes as pinned candidates, and evaluate $\lambda_{\max }\left(\left(G^{S}\right)_{l}\right)$.

Step 2. If pinning condition

$$
\lambda_{\max }\left(\left(G^{s}\right)_{l}\right)<-\frac{a+p}{2 c \gamma}
$$

is satisfied, then go to Step 3; otherwise, $l=l+1$; return to Step 1.

Step 3. According to the number $l$ determined in Step 2, decompose the matrix $G^{s}$ as

$$
G^{s}=\left(\begin{array}{cc}
E & B \\
B^{T} & C
\end{array}\right)
$$

where matrices $E \in \mathbb{R}^{l \times l}, B \in \mathbb{R}^{l \times(N-l)}$, and $C=\left(G^{s}\right)_{l} \in$ $\mathbb{R}^{(N-l) \times(N-l)}$. Choose appropriate $d_{i}, i=1,2, \ldots, l$, such that

$$
d_{i}>\lambda_{\max }\left(E-B C^{-1} B^{T}\right)
$$

is satisfied, end.

According to the above discussion, we present an original frame for designing the intermittent pinning controllers (7). In the next section, we will illustrate effectiveness of our theoretical analysis by numerical examples.

Remark 11. For an undirected network, we have $\operatorname{Deg}_{\text {out }}(i)=$ $\operatorname{Deg}_{\text {in }}(i)(i=1,2, \ldots, N)$ and $G^{s}=G$ due to every node having the same out-degree and in-degree. Therefore, choosing arbitrary $l(1 \leq l \leq N)$ nodes, we always get $\lambda_{\max }\left(\left(G^{s}\right)_{l}\right) \leq 0$ under Assumption 5. Hence, both specifically and randomly pinning strategies are feasible for the synchronization of undirected networks. Meanwhile, we also can find that randomly pinning scheme may not ensure the pinning synchronization of directed network using intermittent controllers, even if a very large coupling strength is adopted.

\section{Numerical Examples}

In this section, we provide a numerical example to verify the effectiveness of the theoretical techniques. Construct a simple directed complex dynamical network with 12 nodes shown in Figure 1. The controlled directed complex network (6) 


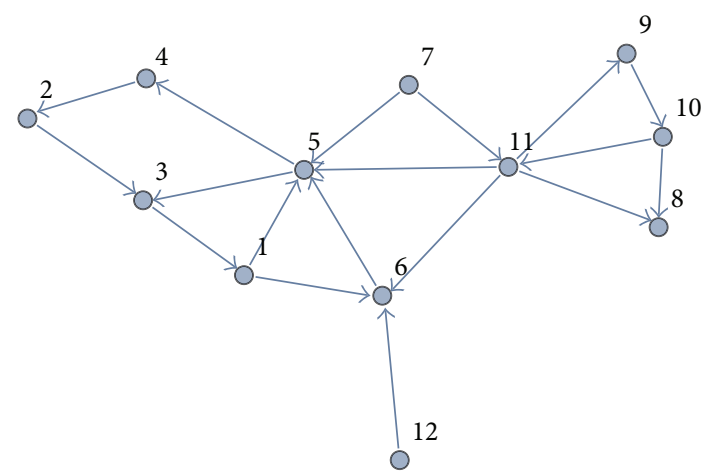

FIgURE 1: Simple directed complex network with 12 nodes.

consisting of 12 identical Chua oscillators with time delayed nonlinearity is described by

$$
\begin{aligned}
\dot{x}_{i}(t)= & f\left(t, x_{i}(t), x_{i}(t-\tau)\right) \\
& +c \sum_{j=1}^{12} g_{i j} \Gamma x_{j}(t)+u_{i}(t), \quad i=1,2, \ldots, 12,
\end{aligned}
$$

where $x_{i}(t)=\left(x_{i 1}(t), x_{i 2}(t), x_{i 3}(t)\right)^{T} \in \mathbb{R}^{3}$ is the state variable of the $i$ th node, coupling strength $c=45$, and time delay $\tau=0.04$. The inner coupling matrix $\Gamma=\operatorname{diag}(1,2,1)$; $G=\left(g_{i j}\right)_{12 \times 12}$ is the coupling configuration matrix of directed complex network described by Figure 1 . Hence, we can get $\gamma=1, \lambda_{\max }\left(G^{s}\right)=0.3560$.

The dynamics of the Chua oscillator is given by

$$
\begin{aligned}
\dot{x}_{i}(t) & =f\left(t, x_{i}(t), x_{i}(t-\tau)\right) \\
& =A x_{i}(t)+g_{1}\left(x_{i}(t)\right)+g_{2}\left(x_{i}(t-\tau)\right),
\end{aligned}
$$

where the matrix

$$
A=\left(\begin{array}{ccc}
-\alpha\left(1+h_{2}\right) & \alpha & 0 \\
1 & -1 & 1 \\
0 & -\eta & -\omega
\end{array}\right)
$$

the nonlinear vector functions

$$
\begin{gathered}
g_{1}\left(x_{i}(t)\right)=\left(-\frac{\alpha}{2}\left(h_{1}-h_{2}\right)\right. \\
\left.\cdot\left(\left|x_{i 1}(t)+1\right|-\left|x_{i 1}(t)-1\right|\right), 0,0\right)^{T}, \\
g_{2}\left(x_{i}(t)\right)=\left(0,0,-\eta \epsilon \sin \left(\nu x_{i 1}(t-\tau)\right)\right)^{T},
\end{gathered}
$$

and $\alpha=10, \eta=19.53, \omega=0.1636, h_{1}=-1.4325$, $h_{2}=-0.7831, \epsilon=0.2$, and $\mu=0.5$. Figure 2 shows the chaotic behavior of time delayed Chua attractor where the initial conditions are given by $x_{i 1}(t)=0.2, x_{i 2}(t)=0.23$,

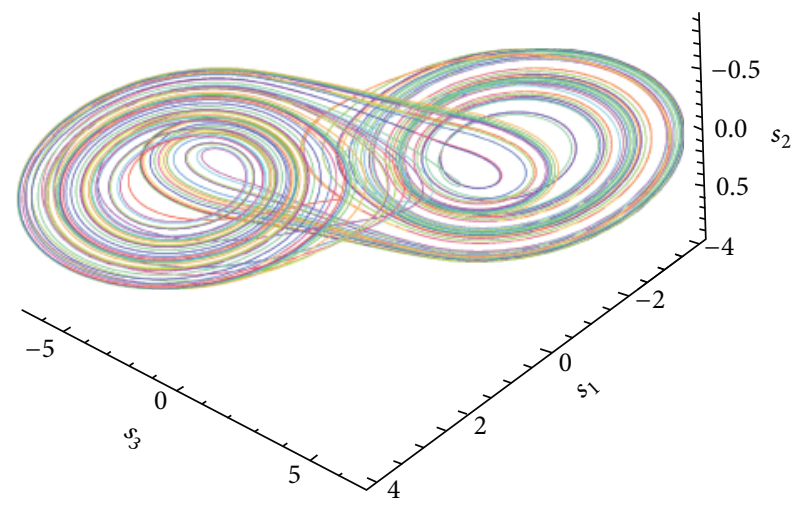

FIgURE 2: Chaotic behavior of delayed Chua attractor.

$x_{i 3}(t)=0.02$, and $t \in[-\tau, 0]$. In addition, we can find two real constant matrices

$$
\begin{aligned}
K & =\left(\begin{array}{ccc}
8.663 & 10 & 0 \\
1 & 1 & 1 \\
0 & 19.53 & 0.1636
\end{array}\right), \\
W & =\left(\begin{array}{ccc}
0 & 0 & 0 \\
0 & 0 & 0 \\
1.953 & 0 & 0
\end{array}\right),
\end{aligned}
$$

such that the nonlinear vector function $f$ in network (26) satisfies Assumption 4. Hence, according to the definitions of parameters $p, q$ in (11), we can get $p=33.53, q=1.953$, where $\varepsilon=1 / 2$

In the following, we can obtain $R_{1}=0.8, R_{2}=0.1$, and $\sigma=26.381$ if the parameters $a=32, b=85, T=0.4$, and $\delta=$ 0.32 are chosen. Correspondingly, the following conditions hold:

$$
\begin{aligned}
a & >q, \\
\lambda_{\text {max }}\left(G^{s}\right) & <\frac{b-p}{2 c \gamma}, \\
\sigma\left(R_{1}-R_{2}\right)-(b+q)\left(1-R_{1}\right) & >0 .
\end{aligned}
$$

Examining the out-degree and in-degree of each node in network described by Figure 1, we rearrange the order of all nodes in accordance with the rule proposed in Section 4. The new order is $7,12,11,1,10,2,4,9,3,5,6,8$. Here, we still denote the rearranged coupling configuration matrix by $G$. Choosing the pinned node $l$ from 1 to 11 and depicting $\lambda_{\max }\left(\left(G^{s}\right)_{l}\right)$, we find that it decreases gradually with increasing of the number $l$ (see Figure 3).

When $l=6$, we have $\lambda_{\max }\left(\left(G^{s}\right)_{6}\right)=-0.9088<-(a+$ p) $/ 2 c \gamma=-0.7281$. Decomposing the matrix $G^{s}$ as the block matrix $G^{S}=\left(\begin{array}{cc}E & B \\ B^{T} & C\end{array}\right)$, we can estimate the lower bound of the pinning feedback gain $d=\lambda_{\max }\left(E-B C^{-1} B^{T}\right)=0.4385$. Hence, we can allocate 6 periodic intermittent controllers to node " $7,12,11,1,10,2$," respectively, and their feedback gains are not less than 0.4385 . Based on above discussion and 


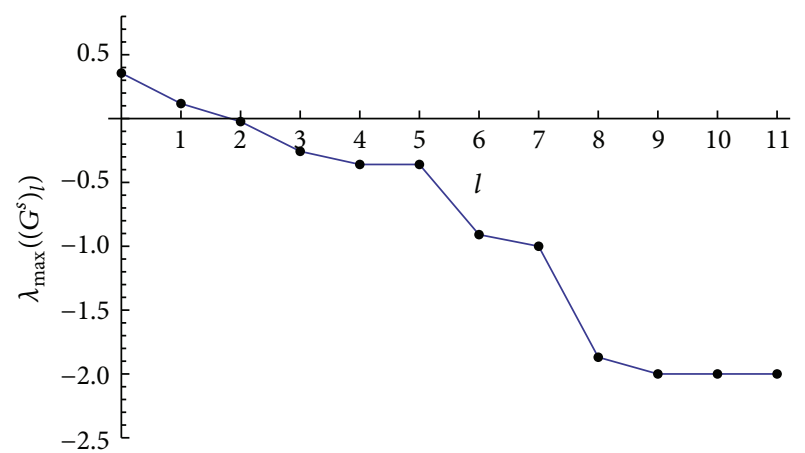

Figure 3: The decline curve of $\lambda_{\max }\left(\left(G^{s}\right)_{l}\right)$ with the number of pinned nodes.

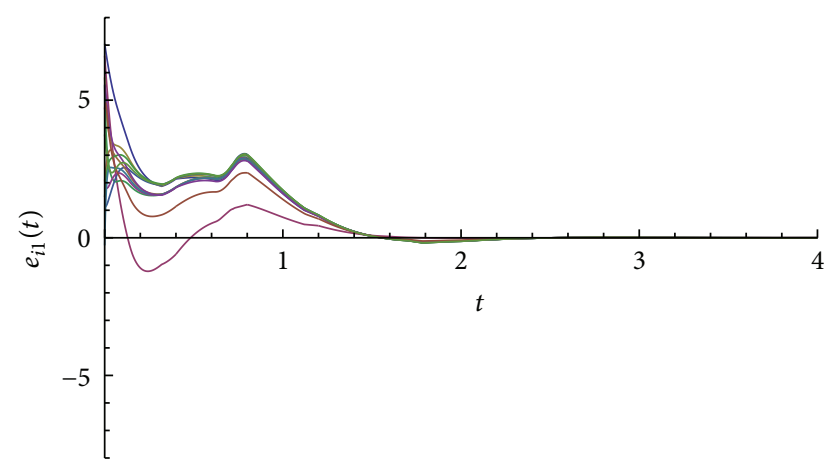

FIGURE 4: Synchronous error $e_{i 1}(t)$ of complex delayed network (26).

Corollary 7, we can realize the pinning synchronization of network (26). In the numerical simulation, we take feedback gains as follows:

$$
\left(d_{1}, \ldots, d_{6}\right)=(2.29,2.34,2.95,2.47,1.28,1.08) .
$$

The initial conditions of $\left(x_{i 1}(0), x_{i 2}(0), x_{i 2}(0)\right) i=1, \ldots, 12$ and $\left(s_{1}(0), s_{2}(0), s_{3}(0)\right)$ are selected randomly on the interval $[-4,4]$. The synchronous errors $e_{i}(t)=x_{i}(t)-s(t), 1 \leq i \leq 12$, are illustrated in Figures 4-6.

\section{Conclusion}

In this paper, the pinning synchronization problem has been investigated for a directed complex network with delayed dynamics. A pinning synchronous criterion based on periodically intermittent control strategy has been proposed, and its applicable versions are also given to illustrate two crucial techniques in pinning control scheme: (i) How many and what type of nodes can be chosen as pinning candidates? (ii) How do we allocate these pinning controllers to network's nodes? Using the proposed algorithm and numerical simulation, it has been shown that pinning synchronization can be reached in a directed complex network by designing periodically intermittent control scheme. Further research direction would include the investigations on delay-inducing synchronization and application of intermittent control on

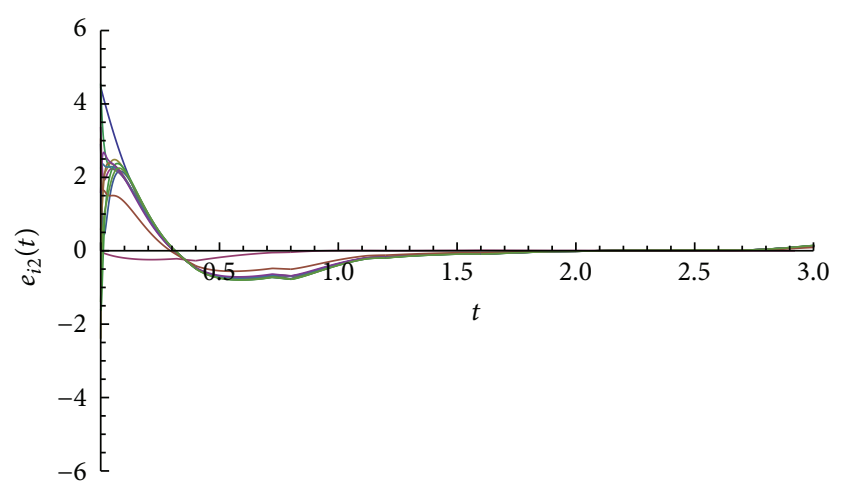

FIGURE 5: Synchronous error $e_{i 2}(t)$ of complex delayed network (26).

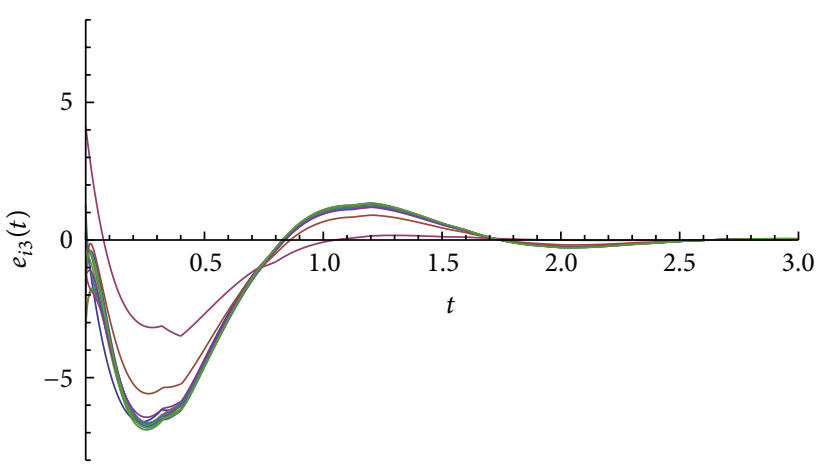

FIGURE 6: Synchronous error $e_{i 3}(t)$ of complex delayed network (26).

consensus of multiagents system (including first- or secondorder) such as those described in $[24,25]$.

\section{Appendix}

\section{Proof of Theorem 6}

Construct the following Lyapunov function:

$$
V(t)=\frac{1}{2} \sum_{i=1}^{N} e_{i}^{T}(t) e_{i}(t)=\frac{1}{2} e^{T}(t)\left(I_{N} \otimes I_{m}\right) e(t),
$$

where $e(t)=\left(e_{1}^{T}(t), e_{2}^{T}(t), \ldots, e_{N}^{T}(t)\right)^{T}$. The derivative of $V$ with respect to $t$ along the trajectory of (9) is as follows:

$$
\begin{aligned}
& \dot{V}(t)=e^{T}(t)\left(I_{N} \otimes I_{n}\right)\left[F(t, x(t), x(t-\tau))-1_{N}\right. \\
& \quad \otimes f(t, s(t), s(t-\tau))]+e^{T}(t)[(c G-D) \otimes \Gamma] e(t),
\end{aligned}
$$

where $t \in[n T, n T+\delta)$ and $n=0,1,2, \ldots$ 
According to Assumption 4, we have

$$
\begin{gathered}
e^{T}(t)\left(I_{N} \otimes I_{n}\right)\left[F(t, x(t), x(t-\tau))-1_{N}\right. \\
\otimes f(t, s(t), s(t-\tau))] \leq \sum_{i=1}^{N} \sum_{r=1}^{m}\left|e_{i r}(t)\right| \\
\cdot \sum_{v=1}^{m}\left(k_{r v}\left|e_{i v}(t)\right|+w_{r v}\left|e_{i v}(t-\tau)\right|\right) \leq \frac{1}{2} \\
\cdot \sum_{i=1}^{N}\left[\sum_{r=1}^{m} \sum_{v=1}^{m}\left(k_{r v}^{2 \varepsilon}+k_{v r}^{2(1-\varepsilon)}+w_{r v}^{2 \varepsilon}\right) e_{i r}^{2}(t)\right. \\
\left.+\sum_{r=1}^{m} \sum_{v=1}^{m} w_{v r}^{2(1-\varepsilon)} e_{i r}^{2}(t-\tau)\right]
\end{gathered}
$$

$$
\begin{aligned}
& \cdot \sum_{i=1}^{N} \sum_{r=1}^{m} e_{i r}^{2}(t-\tau)=\frac{p}{2} e^{T}\left(I_{N} \otimes I_{m}\right) e(t)+\frac{q}{2} e^{T}(t \\
& -\tau)\left(I_{N} \otimes I_{m}\right) e(t-\tau) .
\end{aligned}
$$

Hence,

$$
\begin{aligned}
\dot{V}(t) \leq & -\frac{a}{2} e^{T}(t)\left(I_{N} \otimes I_{m}\right) e(t) \\
& +\frac{q}{2} e^{T}(t-\tau)\left(I_{N} \otimes I_{m}\right) e(t-\tau) \\
& +e^{T}(t)\left[\left(\frac{a+p}{2 \gamma} I_{N}-D+c G^{s}\right) \otimes \Gamma\right] e(t) .
\end{aligned}
$$

By condition (12), we can derive that

$$
\begin{aligned}
\dot{V}(t) & \leq-a V(t)+q V(t-\tau) \\
& \leq-a V(t)+q\left(\sup _{t-\tau \leq \theta \leq t} V(\theta)\right) .
\end{aligned}
$$

Since $a>q$, it follows from Lemma 1 that

$$
V(t) \leq\left(\sup _{n T-\tau \leq \theta \leq n T} V(\theta)\right) \exp (-\sigma(t-n T)),
$$

where $n T \leq t \leq n T+\delta$, and $\sigma$ is the smallest positive real root of equation $a-z-q \exp (z \tau)=0$.

On the other hand, when $t \in[n T+\delta,(n+1) T)$, for every $n=0,1,2, \ldots$, one has

$$
\begin{aligned}
\dot{V}(t) \leq & \frac{b}{2} e^{T}(t)\left(I_{N} \otimes I_{m}\right) e(t) \\
& +\frac{q}{2} e^{T}(t-\tau)\left(I_{N} \otimes I_{m}\right) e(t-\tau) \\
& +e^{T}(t)\left[\left(c G^{s}+\frac{p-b}{2 \gamma} I_{N}\right) \otimes \Gamma\right] e(t) .
\end{aligned}
$$

From condition (12) and definition of $V(t)$, one has

$$
\begin{aligned}
\dot{V}(t) & \leq b V(t)+q V(t-\tau) \\
& \leq b V(t)+q\left(\sup _{t-\tau \leq \theta \leq t} V(\theta)\right) .
\end{aligned}
$$

According to Lemma 2, it yields that

$$
V(t) \leq\left(\sup _{n T+\delta-\tau \leq \theta \leq n T+\delta} V(\theta)\right) \exp (\beta(t-n T-\delta)),
$$

where $\beta=b+q, n T+\delta \leq t \leq(n+1) T$. Next, we estimate $V(t)$ according to (A.7) and (A.10). For $n=0$, it is easy to get

$$
\begin{aligned}
& V(t) \leq\left(\sup _{-\tau \leq \theta \leq 0} V(\theta)\right) \exp (-\sigma t), \quad 0 \leq t<\delta, \\
& V(t) \leq\left(\sup _{\delta-\tau \leq \theta \leq \delta} V(\theta)\right) \exp (\beta(t-\delta)),
\end{aligned}
$$

$$
\delta \leq t<T
$$

Note that $\tau<\delta$; then when $\delta-\tau \leq \theta \leq \delta$, one has $V(\theta) \leq$ $\left(\sup _{-\tau \leq \theta \leq 0} V(\theta)\right) \exp (-\sigma(\delta-\tau))$. Therefore, when $\delta \leq t<T$, it implies that

$$
V(t) \leq\left(\sup _{-\tau \leq \theta \leq 0} V(\theta)\right) \exp (\beta(t-\delta)-\sigma(\delta-\tau)) .
$$

For $n=1$, from (A.7), we can get

$$
\begin{aligned}
V(t) & \leq\left(\sup _{T-\tau \leq \theta \leq T} V(\theta)\right) \exp (-\sigma(t-T)) \\
& \leq\left(\sup _{-\tau \leq \theta \leq 0} V(\theta)\right) \\
& \cdot \exp (\beta(T-\delta)-\sigma(\delta-\tau)-\sigma(t-T)),
\end{aligned}
$$

where $T \leq t<T+\delta$. By (A.10), we have

$$
\begin{aligned}
V(t) & \leq\left(\sup _{T+\delta-\tau \leq \theta \leq T+\delta} V(\theta)\right) \exp (-\sigma(t-T)) \\
& \leq\left(\sup _{-\tau \leq \theta \leq 0} V(\theta)\right) \\
& \cdot \exp \{\beta(T-\delta)+\beta(t-T-\delta)-2 \sigma(\delta-\tau)\},
\end{aligned}
$$

where $T+\delta \leq t<2 T$.

Generally, we have the following estimation of $V(t)$ for any positive integer $n$ by induction. For $n T \leq t<n T+\delta$ and $n=0,1, \ldots$,

$$
\begin{aligned}
& V(t) \leq\left(\sup _{-\tau \leq \theta \leq 0} V(\theta)\right) \\
& \cdot \exp \{-\sigma(t-n T)-n \sigma(\delta-\tau)+n \beta(T-\delta)\} .
\end{aligned}
$$


With respect to $n T+\delta \leq t<(n+1) T$ and $n=0,1, \ldots$,

$$
\begin{aligned}
& V(t) \leq\left(\sup _{-\tau \leq \theta \leq 0} V(\theta)\right) \exp \{\beta(t-n T-\delta) \\
& +n \beta(T-\delta)-(n+1) \sigma(\delta-\tau)\} .
\end{aligned}
$$

Note that if $n T \leq t<n T+\delta$ holds, then $t-\delta<n T \leq t$. Recall the definition of $R_{1}$ and $R_{2}$; inserting $\delta=R_{1} T$ and $\tau=R_{2} T$ into (A.15), one gets that

$$
\begin{aligned}
& V(t) \leq\left(\sup _{-\tau \leq \theta \leq 0} V(\theta)\right) \exp \left\{\beta\left(1-R_{1}\right) t\right. \\
& \left.+\sigma\left(R_{1}-R_{2}\right)(\delta-t)\right\}=\left(\sup _{-\tau \leq \theta \leq 0} V(\theta)\right) \\
& \quad \cdot \exp \left\{-\left[\sigma\left(R_{1}-R_{2}\right)-\beta\left(1-R_{1}\right)\right] t\right. \\
& \left.+\sigma\left(R_{1}-R_{2}\right) \delta\right\} .
\end{aligned}
$$

On the other hand, if $n T+\delta \leq t<(n+1) T$ holds, from (A.16), then we also derive that

$$
\begin{aligned}
& V(t) \leq\left(\sup _{-\tau \leq \theta \leq 0} V(\theta)\right) \exp \left\{\beta t-(n+1) \beta R_{1} T\right. \\
& \left.-(n+1) \sigma\left(R_{1}-R_{2}\right) T\right\} \leq\left(\sup _{-\tau \leq \theta \leq 0} V(\theta)\right) \\
& \cdot \exp \left\{\beta t-\beta R_{1} t-\sigma\left(R_{1}-R_{2}\right) t\right\} \\
& \quad \leq\left(\sup _{-\tau \leq \theta \leq 0} V(\theta)\right) \\
& \cdot \exp \left\{-\left[\sigma\left(R_{1}-R_{2}\right)-\beta\left(1-R_{1}\right)\right] t\right. \\
& \left.+\sigma\left(R_{1}-R_{2}\right) \delta\right\} .
\end{aligned}
$$

By (A.17) and (A.18), for any $t \geq 0$, we always have

$$
\begin{aligned}
& V(t) \leq\left(\sup _{-\tau \leq \theta \leq 0} V(\theta)\right) \\
& \cdot \exp \left\{-\left[\sigma\left(R_{1}-R_{2}\right)-\beta\left(1-R_{1}\right)\right] t\right. \\
& \left.\quad+\sigma\left(R_{1}-R_{2}\right) \delta\right\} .
\end{aligned}
$$

According to definition (A.1) of Lyapunov function $V(t)$, for any $i=1,2, \ldots, N$, one has

$$
\begin{aligned}
& \left\|e_{i}(t)\right\| \leq\left(\sum_{i=1}^{N} e_{i}^{T}(t) e_{i}(t)\right)^{1 / 2} \\
& \quad \leq M \exp \left\{-\frac{1}{2}\left[\sigma\left(R_{1}-R_{2}\right)-\beta\left(1-R_{1}\right)\right] t\right\},
\end{aligned}
$$

where

$$
M=\left(2 \sup _{-\tau \leq \theta \leq 0} V(\theta)\right)^{1 / 2} \exp \left\{\frac{\sigma\left(R_{1}-R_{2}\right) \delta}{2}\right\}>0 .
$$

According to condition (16), we have $\sigma\left(R_{1}-R_{2}\right)-\beta(1-$ $\left.R_{1}\right)>0$. Finally, we conclude that

$$
\lim _{t \rightarrow \infty}\left\|e_{i}(t)\right\|=0, \quad i=1,2, \ldots, N
$$

Thus, we can draw the conclusion that globally asymptotical synchronization of network (6) is achieved by (8). The proof is completed.

\section{Conflict of Interests}

The authors declare that there is no conflict of interests regarding the publication of this paper.

\section{Acknowledgments}

This work was supported by the National Natural Science Foundation of China and the Natural Science Foundation of Honghe University (Grant no. XJ15SX03).

\section{References}

[1] L. M. Pecora and T. L. Carroll, "Synchronization in chaotic systems," Physical Review Letters, vol. 64, no. 8, pp. 821-824, 1990.

[2] J. Cao, R. Sivasamy, and R. Rakkiyappan, "Sampled-data $H_{\infty}$ synchronization of chaotic Lur'e systems with time delay," Circuits, Systems \& Signal Processing, 2015.

[3] J. Cao, R. Rakkiyappan, K. Maheswari, and A. Chandrasekar, "Exponential $H_{\infty}$ filtering analysis for discrete-time switched neural networks with random delays using sojourn probabilities," Science China Technological Sciences, 2016.

[4] D. H. Ji, S. C. Jeong, J. H. Park, S. M. Lee, and S. C. Won, "Adaptive lag synchronization for uncertain complex dynamical network with delayed coupling," Applied Mathematics and Computation, vol. 218, no. 9, pp. 4872-4880, 2012.

[5] W. Guo, "Lag synchronization of complex networks via pinning control," Nonlinear Analysis: Real World Applications, vol. 12, no. 5, pp. 2579-2585, 2011.

[6] X. Liu, T. Chen, J. Cao, and W. Lu, "Dissipativity and quasisynchronization for neural networks with discontinuous activations and parameter mismatches," Neural Networks, vol. 24, no. 10, pp. 1013-1021, 2011.

[7] L. Pan and J. Cao, "Stochastic quasi-synchronization for delayed dynamical networks via intermittent control," Communications in Nonlinear Science and Numerical Simulation, vol. 17, no. 3, pp. 1332-1343, 2012.

[8] Q. Zhou, P. Shi, S. Xu, and H. Li, "Adaptive output feedback control for nonlinear time-delay systems by fuzzy approximation approach," IEEE Transactions on Fuzzy Systems, vol. 21, no. 2, pp. 301-313, 2013.

[9] J. Lu, J. Kurths, J. Cao, N. Mahdavi, and C. Huang, "Synchronization control for nonlinear stochastic dynamical networks: pinning impulsive strategy," IEEE Transactions on Neural Networks and Learning Systems, vol. 23, no. 2, pp. 285-292, 2012.

[10] B. Liu, W. Lu, and T. Chen, "Synchronization in complex networks with stochastically switching coupling structures," IEEE Transactions on Automatic Control, vol. 57, no. 3, pp. 754760, 2012. 
[11] G. Battistelli, J. P. Hespanha, E. Mosca, and P. Tesi, "Modelfree adaptive switching control of time-varying plants," IEEE Transactions on Automatic Control, vol. 58, no. 5, pp. 1208-1220, 2013.

[12] W. Yu, G. Chen, and J. Lü, "On pinning synchronization of complex dynamical networks," Automatica, vol. 45, no. 2, pp. 429-435, 2009.

[13] F. Sorrentino, M. Di Bernardo, F. Garofalo, and G. Chen, "Controllability of complex networks via pinning," Physical Review E, vol. 75, no. 4, Article ID 046103, 2007.

[14] Q. Song and J. Cao, "On pinning synchronization of directed and undirected complex dynamical networks," IEEE Transactions on Circuits and Systems. I. Regular Papers, vol. 57, no. 3, pp. 672-680, 2010.

[15] Q. Song, F. Liu, J. Cao, and W. Yu, "Pinning-controllability analysis of complex networks: an M-matrix approach," IEEE Transactions on Circuits and Systems. I. Regular Papers, vol. 59, no. 11, pp. 2692-2701, 2012.

[16] P. DeLellis, M. D. Bernardo, and F. Garofalo, "Adaptive pinning control of networks of circuits and systems in lur'e form," IEEE Transactions on Circuits and Systems I: Regular Papers, vol. 60, no. 11, pp. 3033-3042, 2013.

[17] V. Mwaffo, P. DeLellis, and M. Porfiri, "Criteria for stochastic pinning control of networks of chaotic maps," Chaos, vol. 24, no. 1, Article ID 013101, 2014.

[18] W. Xia and J. Cao, "Pinning synchronization of delayed dynamical networks via periodically intermittent control," Chaos, vol. 19, no. 1, Article ID 013120, 2009.

[19] X. Liu and T. Chen, "Cluster synchronization in directed networks via intermittent pinning control," IEEE Transactions on Neural Networks, vol. 22, no. 7, pp. 1009-1020, 2011.

[20] S. Cai, J. Hao, Q. He, and Z. Liu, "Exponential synchronization of complex delayed dynamical networks via pinning periodically intermittent control," Physics Letters A, vol. 375, no. 19, pp. 1965-1971, 2011.

[21] Q. Gan, "Exponential synchronization of stochastic CohenGrossberg neural networks with mixed time-varying delays and reaction-diffusion via periodically intermittent control," Neural Networks, vol. 31, pp. 12-21, 2012.

[22] J. Yu, C. Hu, H. Jiang, and Z. Teng, "Exponential lag synchronization for delayed fuzzy cellular neural networks via periodically intermittent control," Mathematics and Computers in Simulation, vol. 82, no. 5, pp. 895-908, 2012.

[23] A. Halanay, Differential Equations: Stability, Oscillations, Time Lags, Academic Press, New York, NY, USA, 1966.

[24] Q. Song, J. Cao, and W. Yu, "Second-order leader-following consensus of nonlinear multi-agent systems via pinning control," Systems \& Control Letters, vol. 59, no. 9, pp. 553-562, 2010.

[25] Q. Song, F. Liu, J. Cao, and W. Yu, "M-matrix strategies for pinning-controlled leader-following consensus in multiagent systems with nonlinear dynamics," IEEE Transactions on Cybernetics, vol. 43, no. 6, pp. 1688-1697, 2013. 


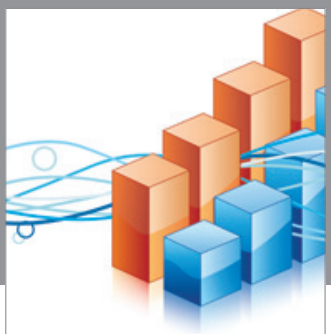

Advances in

Operations Research

vatem alat4

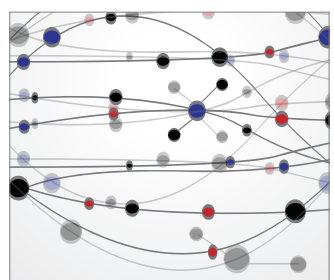

\section{The Scientific} World Journal
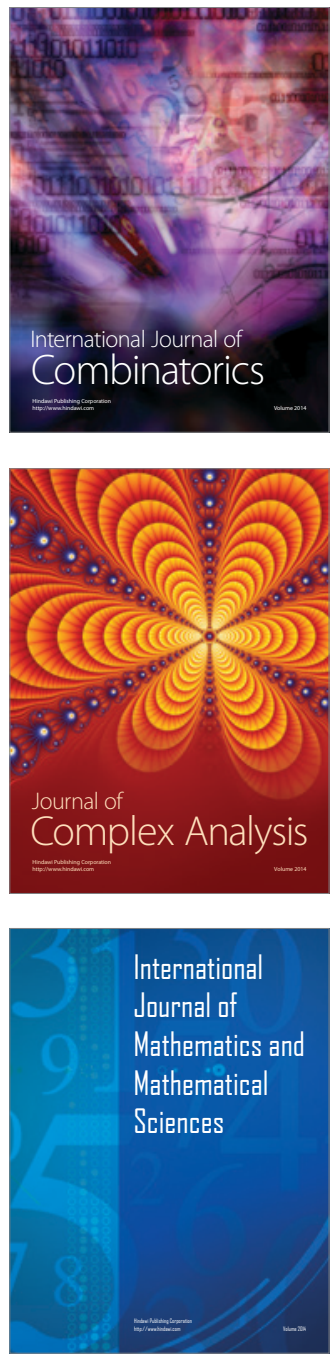
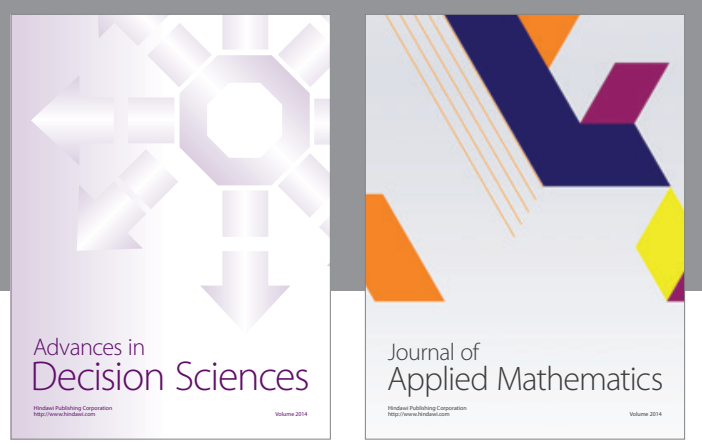

Algebra

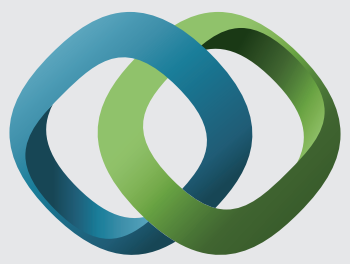

\section{Hindawi}

Submit your manuscripts at

http://www.hindawi.com
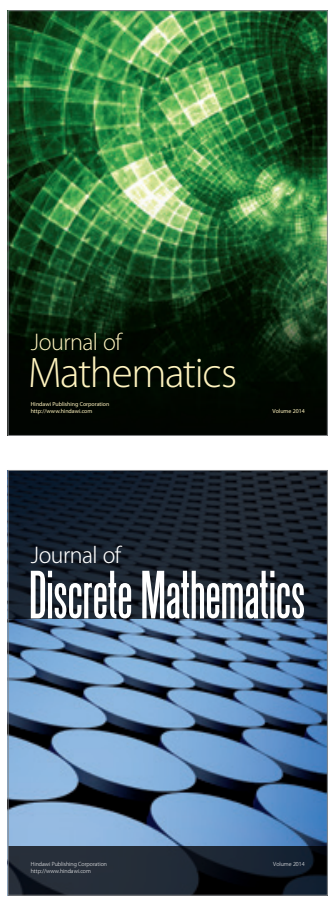

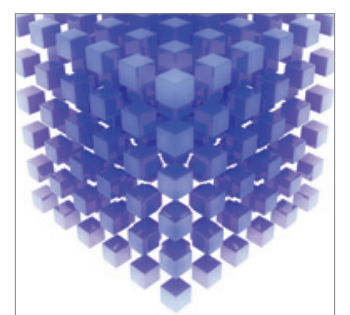

Mathematical Problems in Engineering
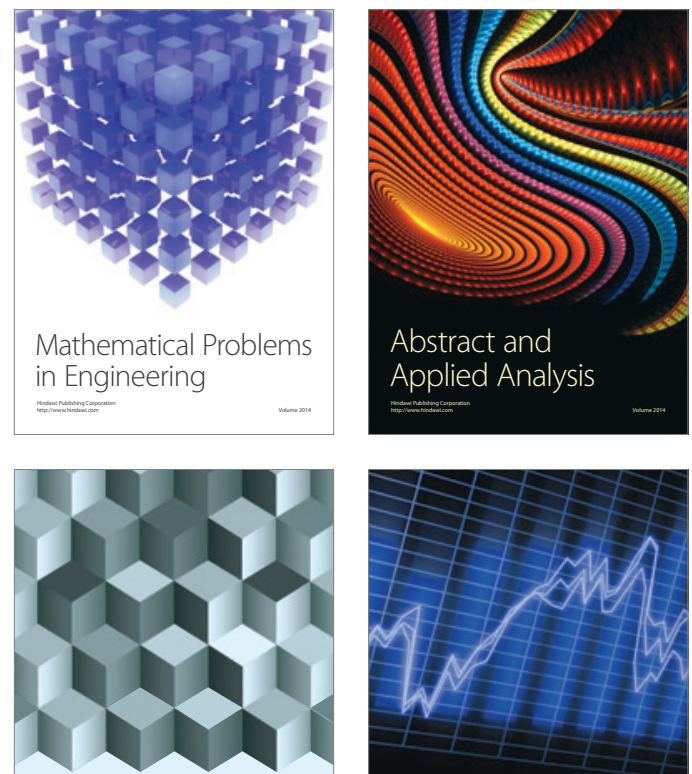

Journal of

Function Spaces

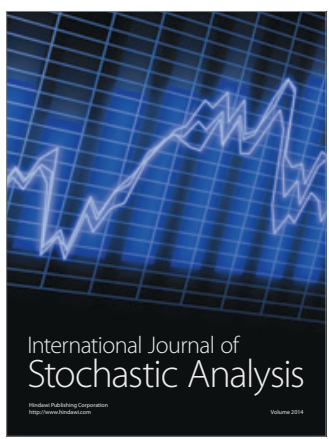

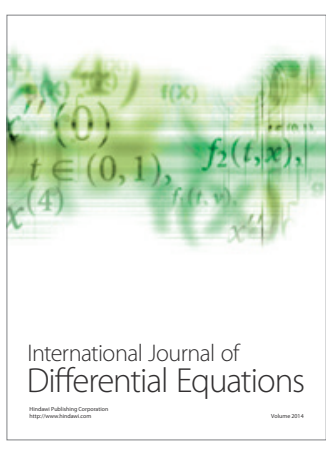
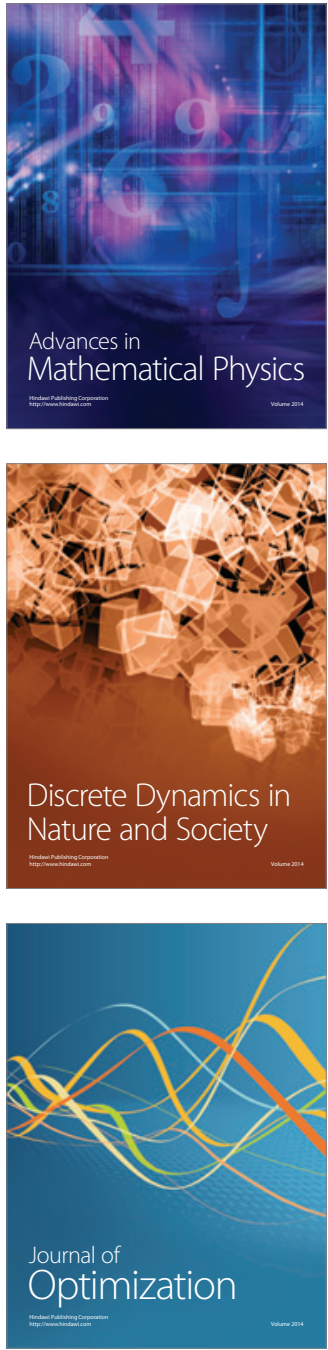